\title{
A radioactive source vehicle recorder based on ARM
}

\author{
Qunying Chen ${ }^{1, a, *}$ \\ ${ }^{1}$ School of ZTE Telecom, Xi'an Peihua University, Xi'an 710125, China \\ achen.20032651@163.com \\ *corresponding author
}

Keywords: Source vehicle recorder, new, ARM, key technology.

\begin{abstract}
This paper introduces a hardware and software structure and key technology of a new radioactive source vehicle recorder based on the 32 bit ARM processor. It is installed in the vehicle which has a radioactive source, it can detecting and recording important datas while the vehicle movement. Starting from the practical application, the ARM with faster operation speed is used as the control center of the system, responsible for system operation, control, management and so on. The key technology of the recorder including LCD display module, voice alarm function, the massive FLASH data memory and USB interface, the host computer communication module, power supply system design. Mainly based on ARM System design, from these modules with the main chip interface discuss the architecture of the whole system.
\end{abstract}

\section{Introduction}

Logging with radioactive source is the main component of nuclear logging instruments. Due to the poor conditions of logging and the around humanities environment influence, inadvertently causing the source lost accident occurred. In order to reduce the number of accidents, countries recommended to install recorder in the vehicle driving on the transport of dangerous goods, synchronize records throughout the process, monitoring the running condition, prevent and avoid possible accidents, implementation of the related emergency plan. For a long time, in order to avoid such accidents, put a lot of manpower and material resources. Some oil fields cost a lot of money to transform the source car into a source car with an alarm system, although this measure is good, but the cost is difficult to promote. Therefore, an intelligent source vehicle recorder fuse with computer technology and communication technology will be an indispensable integrated device of record process 、 displaying and transmitting data to ensure the safe and efficient operation of modern road transportation and dynamic logistics management. But the monitoring facilities and systems for dangerous goods transportation have not been kept up in time.

The integration of modern electronic circuits more and more high, low-end IC has lost the price advantage, while the high-end electronics with its powerful and reliable performance has been widely used. With the wide application of 16/32bit CPU, vehicle integrated information recorder CPU system is gradually from 8bit CPU to a higher bit processor. The powerful function of the latter limited 8 bit CPU development, the graphical interface and dynamic display speed put forward higher requirements for CPU, 16/32bit CPU can normal work at far higher clock frequency than 8bit CPU, one-time data throughput big, processor price decline, making 16/32bit CPU has been widely used in the automotive integrated information recorder.

After the author in-depth analysis of the oil source lost accident case, launched a logging monitoring radioactive source tank monitoring system, It provides an effective and economic tool for the detection of lost source dose, minimizing the loss of the source accidents and once the accident occurs, follow the clues of the missing source . 


\section{System hardware architecture}

\subsection{Master control chip selection}

In order to improve the speed of the MCU, select Enzhike ARM7 64 pin package LPC2119 as the system CPU, in the same crystal frequency faster than the 51 Series MCU more than 40 times, and can increase the performance of friendly humanized interface.LPC2119 is 16/32bit CPU of real-time simulation and tracking, with high speed FLASH memory, can be erased and written at least 100000 times, flash programming time limit $1 \mathrm{~ms}$,can programmable 512 byte, sector erase or chip erase only $400 \mathrm{~ms}$; 128 bit memory interface can achieve up to $60 \mathrm{MHz}$ operating frequency and unique acceleration structure make 32 bit code can run at the maximum clock rate ; ISP and IAP on-chip bootloader; very low power consumption; multiple serial interfaces, including 2 UART, 1 high speed $\mathrm{I}^{2} \mathrm{C}$ interface $(400 \mathrm{kHz})$ and 2 SPI interface; several 32 bit timers; four 10bit ADC with conversion time low to 2.44us; eight 10bit ADC; two CAN bus and up to 12 external interrupt source, make them particularly suitable for industrial control, medical systems, access control and POS machine ; due to a wide range serial communication interface, is also very suitable for communication gateway 、 protocol converter、 embedded software moderm and the application of various other types.

\subsection{Overall system hardware structure}

The vehicle borne radioactive source whole journey monitor is used to whole real-time online monitor the logging vehicle radioactive source existing condition. It is composed of a ray detector, data acquisition box, control box. Radioactive source tank monitoring system by car battery power supply. The ray detector only measur source tank, identification of source species in a tank via an intelligent processor. The CPU will control source storehouse status display and sound light alarm after measured results are processed, and store the important data. When the vehicle back to the base, can authorized to print out datas, such as: the time when power on and power off, the time when source exist and existless.

The system diagram is as follows:

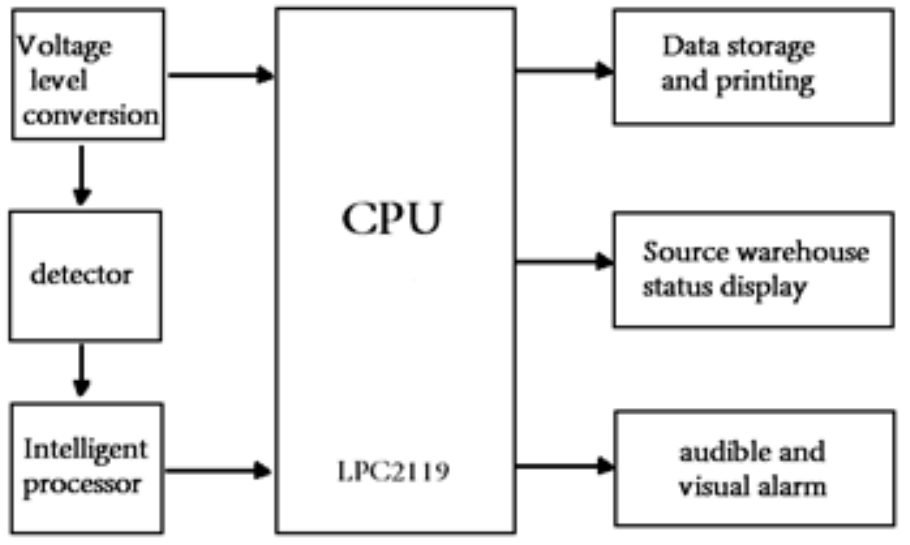

Fig. 1 System diagram

\subsection{Key technology solutions}

\subsubsection{LCD drive control}

This part is the main output window of the system, it is a necessary interface that the system can realize data transmission. This module is responsible for data display, data output and display data synchronization etc.. In order to improve the display mode of the existing liquid crystal display, reduce the lead wire, improve display reliability, we select the 19264 serial LCD display chip , 8 lead wire less than the Parallel port display (the original serial display has 20 pins, Parallel port display has 12 pins), for 19264 chip, we select of blue mold negative display, white backlight, to improve the contrast. 


\subsubsection{Voice alarm function}

This function module is an important module in addition to LCD, unlike the LCD module, through the form of voice to achieve the alarm for the hazardous conditions of the vehicle, to remind the driver Caution!. The module has speech collection and playback functions, can suggesting active, passive, what is the source and field recording, modification of the speech etc.

\subsubsection{FLASH data memory and USB interface}

In order to ensure real-time data storage and read, the module is designed to be responsible for data storage, data and U disk or PC USB interface functions.

The shortcoming of the EEPROM deposit is: the limited storage space, so no storage format, is a set of a group to save, if the data stored behind the wrong, all data will be stored and read data must be wrong, and all the space must be read out, data read is not convenient. So we according to the file format storage, allowing the individual data error. Now the power down storage is use of power detection, often cannot storage, so power check should be avoided.

Flash deposit number idea is as follows:

Calculate with 15 days, storage refresh 4 times per minute, power loss records refreshs once per 15 seconds. Data storage is stored as paged or time-sharing, each boot define a file name for storing data .Data read according to the data file, can reduce error, improve the data read speed.

The AT26DF321 storage structure is $64 * 64 \mathrm{kbyte}$, the way data is stored is shown in the following table:

Table 1 AT26DF321 number of deposits

\begin{tabular}{|c|c|c|c|c|}
\hline A sector & Storage content & Data format & & Remarks \\
\hline 1 & $\begin{array}{c}\text { Record } \\
\text { information }\end{array}$ & Year, month, day & & \\
\hline 2 & Power on & $\begin{array}{c}\text { Year, month, day, hour, minute, } \\
\text { second }\end{array}$ & $64 \mathrm{~K} / 8=8000$ times & Never erase \\
\hline 3 & Power off & $\begin{array}{c}\text { Year, month, hour, minute, } \\
\text { second }\end{array}$ & $64 \mathrm{~K} / 8=8000$ times & Never erase \\
\hline 4 & Spare & $\begin{array}{c}\text { tank 1 2B } \\
\text { tank 2 2B } \\
\text { orlop 2*2=4B }\end{array}$ & $\begin{array}{c}\text { Save valid } \\
\text { records for 15 } \\
\text { days }\end{array}$ \\
\hline $5-63$ & Storage record & & & \\
\hline
\end{tabular}

The remaining $60 * 64 \mathrm{kbyte}$ storage records:

According to 15 day plan: $4 * 64 \mathrm{kB}$ storage space per day, Save every 5 seconds, $8 \mathrm{~B}$ per time (tank 1 , tank 2, orlop), per minute: $8 * 20=160 B$, every half hour: $160 * 30=4800 B(4 K B)$, daily: $4 \mathrm{~K} * 2 * 24=192 \mathrm{kB}<4 * 64 \mathrm{kB}=256 \mathrm{~KB}$. Each half hour for 1 data blocks as $4 \mathrm{~K}$, according to the number of days to boot, cumulative storage 15 days, 15 days later cycle storage.

The data output is changed from RS232 to U disk to export data files

2.3.4 System power section

This part is indispensable for vehicle information recorder, it is responsible for the whole system of power supply, is the center of the system power supply. Require performance and stability is good, otherwise it will cause adverse effects on the system, therefore, its performance directly determines the performance of the system. The system adopts the automobile battery power supply. In the application process found that the power input filter capacitor is inappropriate, capacitance is relatively large impact on the power supply, easy burning fuse. After change power down detection method, can remove the large capacitance.

\section{Software structure}

Software is written in standard C, and is compiled and debugged by ARMADS. The program can be downloaded to the FLASH chip on the motherboard through the JTAG interface.

The program completes the following functions: 
1) Turn on the power supply, the system will enter the self-test, after 30 seconds should be has the successful instructions. The system into the working state, measuring background count rate of source chamber, automatic detection system status.

2) Install the source tank, measure the counting rate, and judge the source of the tank with source or sourceless and the type of source. When there is a source tank put into the source cabin, the safety signal is given. When the source leaves the source tank, the abnormal signal is given and audible and visual alarm are sent out.

3) The storage status of two source tanks in a source warehouse can be displayed at the same time, and the source and the volume of the source chamber are displayed, and process data is stored.

4) Automatically identifies and displays the types of sources for two source tank.

5) Automatically save the records of the source load and unload.

6) Automatically save the time of the monitor power on and off.

7) Authorized to communicate with the computer to view or print the main information on the use of radioactive sources in this operation.

So when the instrument power on and power off, load or take (lost) source events and data detected within the latest 24 hours are recorded by the instrument.

The main flow chart of the program is shown in figure 2-4:

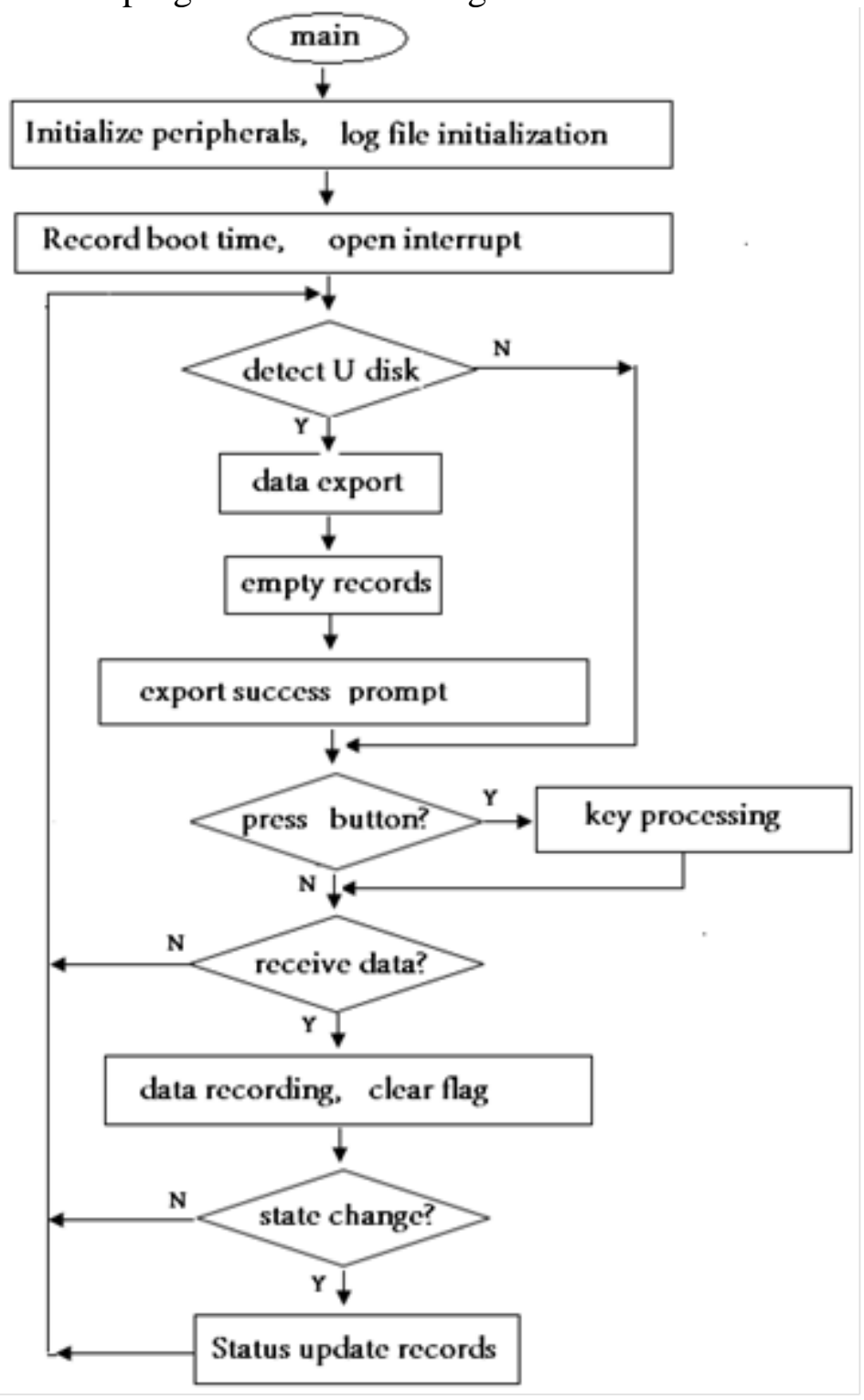

Fig. 2 Flow chart of main program 


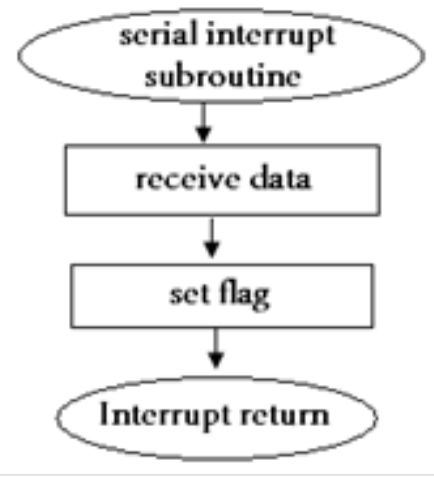

Fig. 3 Serial interrupt subroutine flow chart

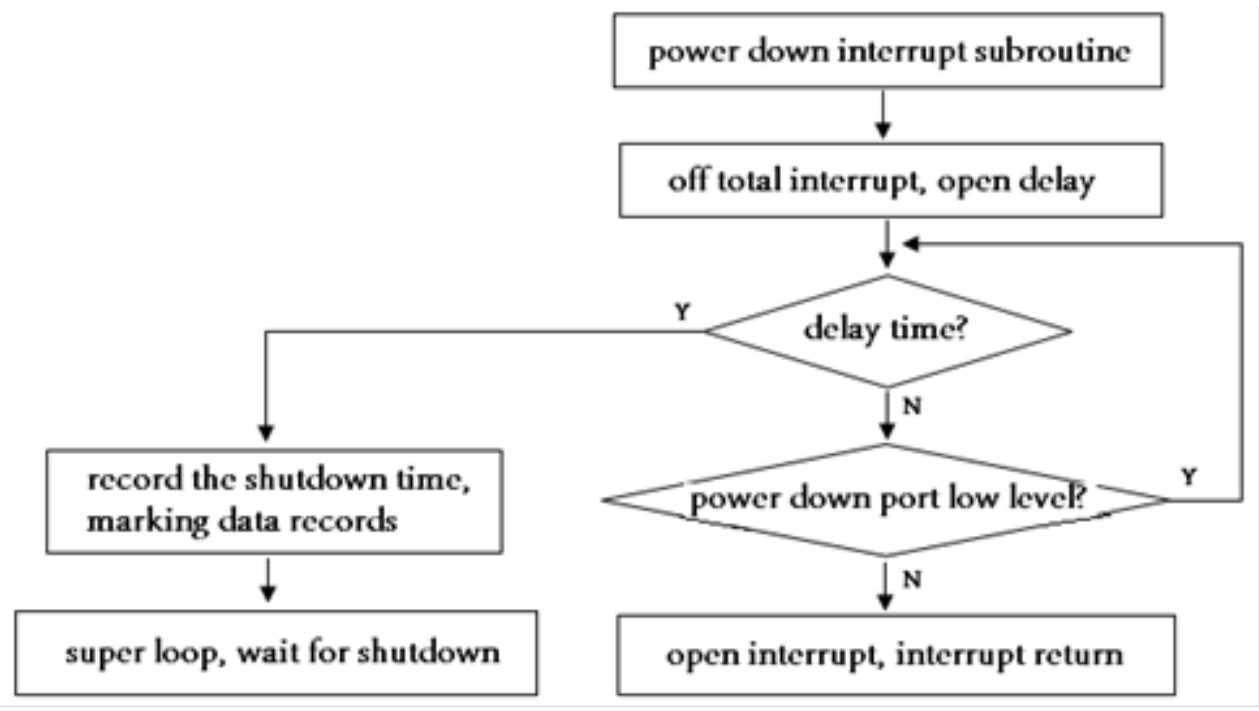

Fig. 4 Flowchart of electric interruption subroutine

\section{Conclusions}

The system realizes the detector from the tank leakage radiation, through the data collecting box processing part for signal processing, send to microprocessor. The results of calculation, judgment to control operation box display and alarm. As long as the source in the source position, will continue to use the instrument Chinese characters display report of radioactive source and real-time status, meanwhile the main information is saved. Managers can not only grasp source's real-time information timely but also through the interface on the computer query and print the historical record. When Lost source accident occurred, would be timely to make a sound, light alarm information, to remind the managers. Once the accident happen, the preservation of historical records may provide useful information for the accident clue.

\section{Acknowledgments}

This work was financially supported by the university level research fund of Xi'an Peihua University (Grant No. PHKT16043).

\section{References}

[1] Zhou li-gong, ARM based embedded system tutorial [M], Beijing, Beihang University press, 2005.

[2] ARM company, ARM Architecture, Reference, Manual [Z].2000. 
[3] General Administration of quality supervision, inspection and quarantine.GB/T 19056-2003[S]. automotive travel data recorder, 2003.

[4] Zhou li-gong, ARM embedded MiniGUI application development and preliminary examples [M], Beijing, Beihang University press, 2005.

[5] Du Chun-lei, ARM architecture and programming, Beijing, Tsinghua University press, 2003.

[6] Wang Shao-chun, Automatic inspection technology, Beijing, Metallurgical Industry Press, 1995. 